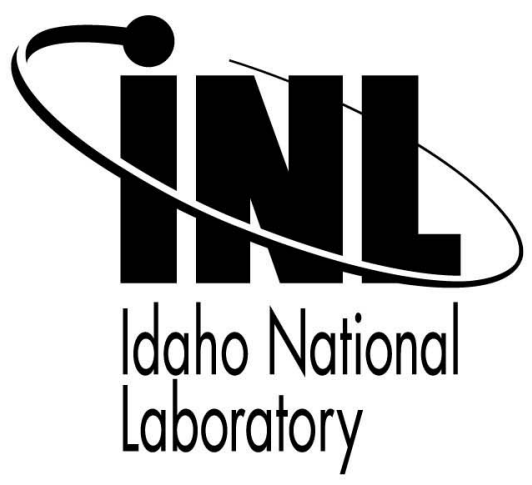

\title{
Modeling Strategies for Unsteady Turbulent Flows in the Lower Plenum of the VHTR
}

\section{OECD/NEA/IAEA Workshop on the Benchmarking of CFD Codes for Application to Nuclear Reactor Safety (CFD4NRS)}

\author{
Richard W. Johnson
}

September 2006
This is a preprint of a paper intended for publication in a journal or proceedings. Since changes may be made before publication, this preprint should not be cited or reproduced without permission of the author. This document was prepared as an account of work sponsored by an agency of the United States Government. Neither the United States Government nor any agency thereof, or any of their employees, makes any warranty, expressed or implied, or assumes any legal liability or responsibility for any third party's use, or the results of such use, of any information, apparatus, product or process disclosed in this report, or represents that its use by such third party would not infringe privately owned rights. The views expressed in this paper are not necessarily those of the United States Government or the sponsoring agency. 


\title{
MODELING STRATEGIES FOR UNSTEADY TURBULENT FLOWS IN THE LOWER PLENUM OF THE VHTR
}

\author{
Richard W. Johnson \\ Idaho National Laboratory, USA
}

\begin{abstract}
Validation simulations are presented for turbulent flow in a staggered tube bank, geometry similar to the lower plenum of a gas-cooled high temperature reactor. Steady 2D RANS results are compared to unsteady 2D RANS results and experiment. The unsteady calculations account for the fact that nonturbulent fluctuations (due to vortex-shedding) are present in the flow. The unsteady computations are shown to predict the mean variables and the total shear stress quite well. Previous workers' results indicate that 3D simulations are needed to obtain reasonable agreement; present results indicate $2 \mathrm{D}$ is sufficient. Best practices are based on requirements for the ASME Journal of Fluids Engineering.
\end{abstract}

\section{Introduction}

The final design of the very high temperature reactor (VHTR) of the fourth generation of nuclear power plants has not been established. The VHTR may be either a block or pebble bed type. However, a conceptual design of a gas-cooled VHTR, based on the General Atomics GT-MHR, does exist and is called the block VHTR reference design, MacDonald et al [1], General Atomics [2]. The present studies are based on the block VHTR reference design. In this design, the flow in the lower plenum will be introduced by dozens of turbulent jets issuing into a large crossflow that must negotiate dozens of cylindrical columns as it flows toward the exit duct. The jets will be at various temperatures due to the radial variation of the core power density. It is important that the coolant be well mixed as it enters the power conversion unit to ensure proper operation and long life of the power machinery. Hence, it is important to accurately model the flow and mixing of the coolant in the lower plenum and exit duct. Figure 1 provides a plan view of half a cross-section of the lower plenum of the reference VHTR. Contours indicate turbulence intensity ( $\mathrm{red}=$ high, blue $=$ low) in this preliminary simulation. As shown, the coolant will have to negotiate a forest of support columns as it flows to the exit duct.

Accurate flow modeling involves determining suitable modeling strategies including the fineness of the grid needed, iterative convergence tolerance, numerical discretization method used, whether the flow is steady or unsteady, and the turbulence model and wall treatment employed. It also involves validation of the computer code and turbulence model against a series of separate and combined flow phenomena and selection of the data used for the validation. In view of the geometry of the lower plenum of the reference VHTR, the flow will be similar to flow in tube banks. The first flow employed for validation studies for normal reactor operation in the VHTR lower plenum is an isothermal incompressible flow in a tube bundle. It will be shown that the flow physics should be understood and simulated properly to be able to achieve validation. Additional validation work will need to be performed against other flow phenomena that will occur in the reactor to fully validate the CFD tools. 


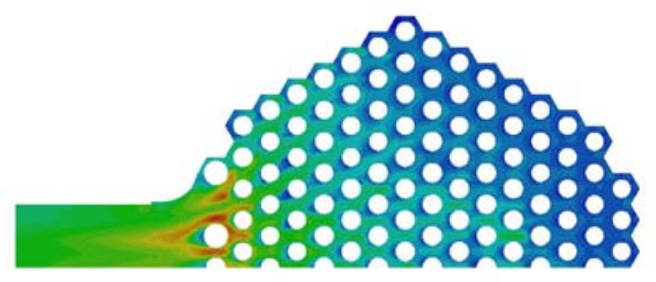

Figure 1. Plan view of a cross-section of a model of the lower plenum for the reference VHTR showing the cylindrical support columns around which the coolant must flow to reach the exit duct; the contours are for turbulence intensity which indicate that the highest levels are near the outlet.

\section{Modeling Flow in a Tube Bundle}

The data of Simonin and Barcouda $[3,4]$ for turbulent flow in a staggered tube bank are used for validation studies. The 1988 reference for Simonin and Barcouda are for the same apparatus as the 1986 data with measurements extended up to the sixth row. Both references are given along with the data on the ERCOFTAC ${ }^{\text {a }}$ database. The data of Simonin and Barcouda (referred to as S\&B hereafter) were used in a comparison study for turbulence models for the 2nd and 3rd ERCOFTAC-IAHR workshops $[5,6]$. The data are provided for comparing turbulence model results and have been used for comparison with numerical simulations by, e.g. Benhamadouche and Laurence [7], Rollet-Miet et al [8], Hassan and Barsamian [9], and Moulinec et al [10]. The S\&B data are given as stationary and the simulations of the workshop participants [5,6] were also stationary [7]. The commercial CFD code FLUENT [11] is used in the present study for the numerical simulations.

\section{Stationary flow modeling}

The flow in the lower plenum of the reference VHTR has been determined to be incompressible. Flow in the tube banks of $\mathrm{S} \& \mathrm{~B}$ is incompressible and isothermal. The continuity and Navier-Stokes equations for isothermal, incompressible flow, given in tensor notation, are (Wilcox [12]):

$$
\begin{aligned}
& \frac{\partial u_{i}}{\partial x_{i}}=0 \\
& \rho \frac{\partial u_{i}}{\partial t}+\rho \frac{\partial u_{i} u_{j}}{\partial x_{j}}=-\frac{\partial p}{\partial x_{i}}+\frac{\partial}{\partial x_{j}}\left[\mu\left(\frac{\partial u_{i}}{\partial x_{j}}+\frac{\partial u_{j}}{\partial x_{i}}\right)\right]+\rho f_{i}
\end{aligned}
$$

where $u_{i}$ is the velocity vector, $p$ is pressure, $\rho$ is density, $\mu$ is the dynamic viscosity, $t$ is time, $f$ is body force and the $x_{i}$ are coordinates. The traditional approach to solving these equations for turbulent flow is to perform Reynolds averaging of the equations. Turbulence itself is three-dimensional and unsteady. However, if the only unsteady motions are turbulent, the flow is said to be stationary, that is, the mean flow is steady. In such a case, Reynolds averaging means to perform a time average of the flow equations. The length of the time average should be sufficient to ensure that the mean quantities so defined do not vary for longer intervals. Reynolds averaging is preceded by a decomposition of the instantaneous values of velocity and pressure into a mean and a turbulent fluctuating quantity, e.g.

\footnotetext{
${ }^{\mathrm{a}}$ http://cfd.me.umist.ac.uk/ercoftac/
} 


$$
u_{i}=U_{i}+u_{i}^{\prime}
$$

After decomposition is applied, the describing equations are time-averaged to yield the Reynoldsaveraged continuity and Navier-Stokes (RANS) equations. With an overbar representing a timeaveraged quantity, these equations are:

$$
\begin{aligned}
& \frac{\partial U_{i}}{\partial x_{i}}=0 \\
& \rho \frac{\partial U_{i}}{\partial t}+\rho \frac{\partial U_{i} U_{j}}{\partial x_{j}}=-\frac{\partial P}{\partial x_{i}}+\frac{\partial}{\partial x_{j}}\left[\mu\left(\frac{\partial U_{i}}{\partial x_{j}}+\frac{\partial U_{j}}{\partial x_{i}}\right)-\rho \overline{u_{i}^{\prime} u_{j}^{\prime}}\right]+\rho f_{i}
\end{aligned}
$$

The time-dependent term on the left-hand side is retained for convenience, though it is zero for a stationary flow. The new term $\rho \overline{u_{i}^{\prime} u_{j}^{\prime}}$, the Reynolds stresses, has been moved to the right side and joined with the diffusion term. This new term represents the action of turbulent fluctuations which could be described as 'turbulent diffusion.' In order to be able to solve the RANS equations, the Reynolds stresses must be modeled using a turbulence model. Though there have been scores of turbulence models developed over the past century, none has been shown to be universally accurate.

The most sophisticated approach using the RANS equations is a Reynolds stress model (RSM), which solves differential transport equations for each Reynolds stress component. Such transport equations are derived from the Navier-Stokes equations, Wilcox [12]. These equations include some terms that are exact and some that must be modeled. Because a separate equation is solved for each Reynolds stress, the model is, in general, anisotropic in terms of the normal Reynolds stresses, unlike eddy viscosity models. That is, $\overline{u^{\prime 2}}, \overline{v^{\prime 2}}$ and $\overline{w^{\prime 2}}$ are not equal, which, in fact, is the case for the data of $\mathrm{S} \& \mathrm{~B}$. However, an RSM requires considerable computation time because from five to seven differential equations for the turbulence quantities must be solved, depending upon dimensionality.

\section{Nonstationary flow modeling}

Hassan and Barsamian [9] indicate that the flow in staggered tube bundles reported by S\&B is a nonstationary turbulent flow. Benhamadouche and Laurence [7] also compute the flow as a nonstationary turbulent flow. That is, the flow is reported to have nonturbulent fluctuations; these fluctuations are associated with vortex-shedding from the tubes. The Reynolds-averaging of the Navier-Stokes equations should then be designed to separate the coherent fluctuations from the turbulent fluctuations; the latter are then modeled with a RANS turbulence model. Turbulence models are not designed to model the effects of coherent fluctuations. This is in contrast to large-eddy simulation (LES) and direct numerical simulation (DNS), which compute directly all fluctuations that are larger than the filter/grid. A RANS turbulence model can be used in an unsteady calculation for a nonstationary turbulent flow. Such a calculation is denoted an unsteady RANS or URANS calculation, Hanjalic [13].

For a URANS calculation, the flow equations should be ensemble-averaged. Theoretically, an ensemble average is applied to experimental data by running the experiment a large number of times, taking data at selected points and at selected times. The data for a given point at a specified time from

each of the tests are averaged to obtain the ensemble average. The difference between the ensemble 
average and the instantaneous velocity are the turbulent fluctuations. The Reynolds stresses thus obtained still vary in time as does the ensemble-averaged velocity.

If the coherent unsteadiness is a well-behaved periodic unsteadiness, the flow may be phaseaveraged, where data are taken at the same time in each cycle to obtain a collection of data which can then be averaged. Hence, phase averaging is a special case of ensemble averaging. In the case of the latter, the ensemble mean quantities must be repeatable from the initial condition, but are not necessarily periodic. The ensemble-averaged equations are still referred to as Reynolds-averaged. First, a Reynolds decomposition is performed:

$$
u=\langle u\rangle+u^{\prime}
$$

where $\langle u\rangle$ is the ensemble mean quantity and $u^{\prime}$ the turbulent fluctuation. Figure 2 illustrates the instantaneous, ensemble-averaged and turbulent fluctuating velocities of Eq. (6) for a nonstationary turbulent flow using simple hand-drawn curves.

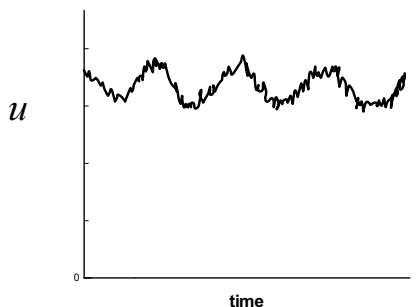

(a)

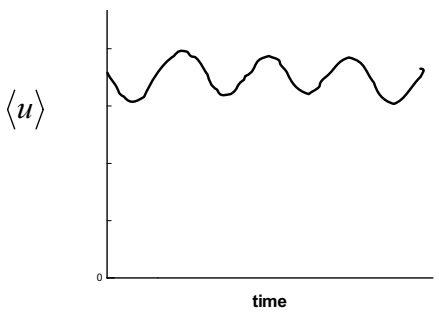

(b)

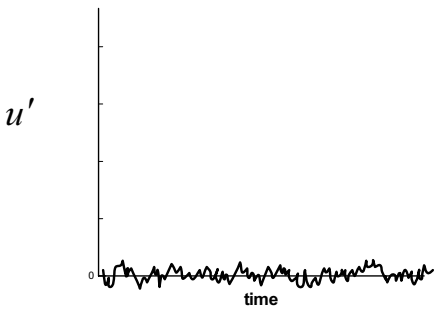

(c)

Figure 2. The instantaneous velocity $u$ for a point in space over a period of time is illustrated in (a), along with (b) the ensemble average $\langle u\rangle$ of the signal and (c) the turbulent fluctuating component $u^{\prime}$.

After the dependent variables in the describing equations are decomposed, Eq. (6), the equations are ensemble-averaged to obtain:

$$
\begin{aligned}
& \frac{\partial\left\langle u_{i}\right\rangle}{\partial x_{i}}=0 \\
& \rho \frac{\partial\left\langle u_{i}\right\rangle}{\partial t}+\rho \frac{\partial\left\langle u_{i}\right\rangle\left\langle u_{j}\right\rangle}{\partial x_{j}}=-\frac{\partial\langle p\rangle}{\partial x_{i}}+\frac{\partial}{\partial x_{j}}\left[\mu\left(\frac{\partial\left\langle u_{i}\right\rangle}{\partial x_{j}}+\frac{\partial\left\langle u_{j}\right\rangle}{\partial x_{i}}\right)-\rho\left\langle u_{i}^{\prime} u_{j}^{\prime}\right\rangle\right]+\rho f_{i}
\end{aligned}
$$

where $\rho\left\langle u_{i}^{\prime} u_{j}^{\prime}\right\rangle$ is the (time-varying) Reynolds stress tensor. Equations $(7,8)$ are those solved for unsteady flow in the present study.

The ensemble-averaged quantities in Eqs. $(7,8)$ may additionally be time-averaged (with a time interval long enough to adequately average all coherent fluctuations) with a secondary decomposition:

$$
\langle u\rangle=U+\widetilde{u}
$$


where $U$ is the time-averaged quantity and $\tilde{u}$ is the coherent fluctuating velocity. Figure 3 illustrates the concept of the time- and ensemble-averaged nonturbulent quantities, Eq. (9). FLUENT [11] allows this type of calculation and refers to it as the 'mean velocity.' The mean velocity is obtained by averaging the velocity at a point over a period of time. If the flow exhibits both turbulent and nonturbulent unsteadiness, then this averaging can be seen as the time average of the ensemble average or just a long-time average.

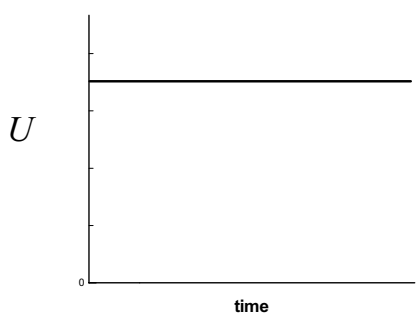

(a)

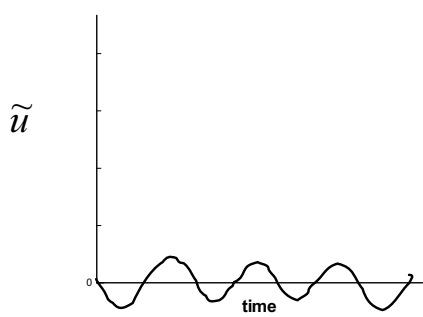

(b)

Figure 3. The (a) time- and ensemble- averaged velocity and (b) the coherent (nonturbulent) fluctuating velocity.

The purpose for performing this second (time) averaging is that some data provided by experimentalists, such as the $\mathrm{S} \& \mathrm{~B}$ data, may have been processed with long-time averaging, rather than by ensemble-averaging. By performing just a long-time average of the experimental data, the turbulent fluctuations are combined with the coherent fluctuations such that only their sum is provided. Upon performing the second decomposition, Eq. (9), and then time-averaging the equations, one arrives at the following equations:

$$
\begin{aligned}
& \frac{\partial U_{i}}{\partial x_{i}}=0 \\
& \rho \frac{\partial U_{i} U_{j}}{\partial x_{j}}=-\frac{\partial P}{\partial x_{i}}+\frac{\partial}{\partial x_{j}}\left[\mu\left(\frac{\partial U_{i}}{\partial x_{j}}+\frac{\partial U_{j}}{\partial x_{i}}\right)-\rho \overline{\widetilde{u}_{i} \widetilde{u}_{j}}-\rho \overline{\left\langle u_{i}^{\prime} u_{j}^{\prime}\right\rangle}\right]+\rho f_{i}
\end{aligned}
$$

where $\rho \overline{\widetilde{u}_{i} \widetilde{u}_{j}}$ is the time average of coherent fluctuations and $\rho \overline{\left\langle u_{i}^{\prime} u_{j}^{\prime}\right\rangle}$ is the time-average of the Reynolds stress tensor. If we had performed a long time average to the instantaneous equations such that all fluctuations were captured, we would have obtained Eqs. (4) and (5) with the correlation term including both coherent and turbulent fluctuations. Denoting the velocity fluctuation, whether turbulent or coherent by $u_{i}^{\prime \prime}$ and considering Eq. (11), we can write

$$
\rho \overline{u_{i}^{\prime \prime} u_{j}^{\prime \prime}}=\rho \overline{\widetilde{u}_{i} \widetilde{u}_{j}}+\rho \overline{\left\langle u_{i}^{\prime} u_{j}^{\prime}\right\rangle}
$$

We see that the total correlation obtained incorporating all fluctuations is equal to the sum of two separate correlations, one for the coherent fluctuations and one for the turbulent fluctuations. The first term on the right hand side of Eq. (12) can be and is computed when using a URANS approach; the second term (Reynolds stress) is obtained via the turbulence model and can be added to the first term. 
A URANS calculation using FLUENT can be made of the flow of Simonin and Barcouda. FLUENT provides the time- and ensemble-averaged mean quantities (or just long-time averaged) as well as root-mean-square (rms) values of the fluctuations. If a RANS turbulence model is used to represent the effects of turbulence, the fluctuations computed in the unsteady simulation (the rms values) will be nonturbulent. These values must be squared to obtain the coherent term in Eq. (12). To obtain the time-average of the Reynolds stresses with FLUENT, a 'user-defined function' (UDF) must be written. Also, coherent cross-correlations $(i \neq j)$ must be obtained using a UDF because FLUENT does not provide these. To obtain the cross-correlation for the coherent fluctuations, one can write:

$$
\overline{\langle u\rangle\langle v\rangle}=\overline{(U+\widetilde{u})(V+\widetilde{v})}=\overline{U V+\widetilde{v} U+\widetilde{u} V+\widetilde{u} \widetilde{v}}=U V+\overline{\widetilde{u} \widetilde{v}}
$$

since the time average of a coherent fluctuating component is zero. From Eq. (13), the coherent fluctuating correlation can be obtained by subtracting the product $U V$ from the time-average of the product of the ensemble-averaged velocities (computed in the UDF).

\section{Turbulence Model}

The computations reported herein are made using the Reynolds stress turbulence model available in FLUENT [11]. The FLUENT RSM model has several options, including the option for computing an extra equation for the turbulent kinetic energy to obtain boundary conditions for the Reynolds stresses. This option is a default option but is disabled as it seems inconsistent with using $\mathrm{k}$ computed with the RSM; it was also found to prevent convergence to $10^{-6}$ tolerance, probably because of the inconsistency. Other than the aforementioned option, all other default options are used. The incompressible RSM in FLUENT used in the present study is based on the Reynolds stress transport equation given as

$$
\frac{\partial}{\partial t}\left(\rho\left\langle u_{i}^{\prime} u_{j}^{\prime}\right\rangle\right)+C_{i j}=D_{T, i j}+\frac{\partial}{\partial x_{k}}\left(\mu \frac{\partial}{\partial x_{k}}\left\langle u_{i}^{\prime} u_{j}^{\prime}\right\rangle\right)+P_{i j}+\Phi_{i j}-\varepsilon_{i j}
$$

where

$$
C_{i j}=\frac{\partial}{\partial x_{k}}\left(\rho\left\langle u_{k}\right\rangle\left\langle u_{i}^{\prime} u_{j}^{\prime}\right\rangle\right) \text { and } P_{i j}=-\rho\left(\left\langle u_{i}^{\prime} u_{k}^{\prime}\right\rangle \frac{\partial\left\langle u_{j}\right\rangle}{\partial x_{k}}+\left\langle u_{j}^{\prime} u_{k}^{\prime}\right\rangle \frac{\partial\left\langle u_{i}\right\rangle}{\partial x_{k}}\right) \text {. }
$$

The turbulent diffusion and pressure-strain terms are modeled, respectively, as

$$
D_{T, i j}=-\frac{\partial}{\partial x_{k}}\left(\frac{\mu_{t}}{\sigma_{k}} \frac{\partial\left\langle u_{i}^{\prime} u_{j}^{\prime}\right\rangle}{\partial x_{k}}\right) \text { and } \Phi_{i j}=\Phi_{i j, 1}+\Phi_{i j, 2}+\Phi_{i j, w}
$$

where

$$
\Phi_{i j, 1}=-C_{1} \rho \frac{\varepsilon}{k}\left(\left\langle u_{i}^{\prime} u_{j}^{\prime}\right\rangle-\frac{2}{3} \delta_{i j} k\right), \quad \Phi_{i j, 2}=-C_{2}\left[\left(P_{i j}-C_{i j}\right)-\frac{2}{3} \delta_{i j}(P-C)\right] \text { and }
$$




$$
\begin{aligned}
\Phi_{i j, w} & =C_{1}^{\prime} \frac{\varepsilon}{k}\left(\left\langle u_{k}^{\prime} u_{m}^{\prime}\right\rangle n_{k} n_{m} \delta_{i j}-\frac{3}{2}\left\langle u_{i}^{\prime} u_{k}^{\prime}\right\rangle n_{j} n_{k}-\frac{3}{2}\left\langle u_{j}^{\prime} u_{k}^{\prime}\right\rangle n_{i} n_{k}\right) \frac{k^{3 / 2}}{C_{\ell} \varepsilon d} \\
& +C_{2}^{\prime}\left(\Phi_{k m, 2} n_{k} n_{m} \delta_{i j}-\frac{3}{2} \Phi_{i k, 2} n_{j} n_{k}-\frac{3}{2} \Phi_{j k, 2} n_{i} n_{k}\right) \frac{k^{3 / 2}}{C_{\ell} \varepsilon d}
\end{aligned}
$$

with $\sigma_{\mathrm{k}}=0.82, C_{1}=1.8, C_{2}=0.6, P=1 / 2 P_{k k}, C=1 / 2 C_{k k}$ (summation over repeated indices), $C_{1}{ }^{\prime}=0.5$, and $C_{2}{ }^{\prime}=0.3 . n_{k}$ is the $x_{k}$ component of the unit normal to the wall, $d$ is the normal distance to the wall, and $C_{\ell}=C_{\mu}^{3 / 4} / \kappa$, where $C_{\mu}=0.09, \mu_{t}=\rho C_{\mu} k^{2} / \varepsilon$, and $\kappa=0.4187$. The turbulent kinetic energy is given as $1 / 2\left\langle u_{i}^{\prime} u_{i}^{\prime}\right\rangle$ and the dissipation rate tensor $\varepsilon_{i j}=2 / 3 \delta_{i j} \rho \varepsilon$ for incompressible flow. The scalar dissipation rate $\varepsilon$ is found by solving its transport equation:

$$
\frac{\partial(\rho \varepsilon)}{\partial t}+\frac{\partial\left(\rho \varepsilon u_{i}\right)}{\partial x_{i}}=\frac{\partial}{\partial x_{j}}\left[\left(\mu+\frac{\mu_{t}}{\sigma_{\varepsilon}}\right) \frac{\partial \varepsilon}{\partial x_{j}}\right]+C_{\varepsilon 1} \frac{1}{2} P_{i i} \frac{\varepsilon}{k}-C_{\varepsilon 2} \rho \frac{\varepsilon^{2}}{k}
$$

where $\sigma_{\varepsilon}=1.0, C_{\varepsilon 1}=1.44$ and $C_{\varepsilon 2}=1.92$.

The wall treatment used for all of the computations reported is the standard wall function approach [11] wherein the mean velocity in the wall-adjacent cell is calculated as

$$
U^{*}= \begin{cases}\frac{1}{\kappa} \ln \left(E y^{*}\right) & \mathrm{y}^{*}>11.225 \\ \mathrm{y}^{*} & \mathrm{y}^{*}<11.225\end{cases}
$$

where

$$
U^{*} \equiv \frac{U_{P} C_{\mu}^{1 / 4} k_{P}^{1 / 2}}{\tau_{w} / \rho}, \text { and } y^{*} \equiv \frac{\rho y_{P} C_{\mu}^{1 / 4} k_{P}^{1 / 2}}{\mu}
$$

and $\kappa=0.4187, E=9.793, U_{P}, k_{P}$ and $y_{P}$ are the mean velocity, turbulence kinetic energy and wall distance at point $\mathrm{P}$ and $\tau_{w}$ is the wall shear stress. However, the near-wall nodes around the circumference of the tubes for the present calculations are within a $\mathrm{y}^{*}$ value of 7 , well within the viscous sublayer. The boundary values for the Reynolds stresses are given as [11]

$$
\frac{\left\langle u_{\tau}^{\prime 2}\right\rangle}{\tau_{w} / \rho}=5.1, \frac{\left\langle u_{\eta}^{\prime 2}\right\rangle}{\tau_{w} / \rho}=1.0, \frac{\left\langle u_{\lambda}^{\prime 2}\right\rangle}{\tau_{w} / \rho}=2.3 \text {, and } \frac{-\left\langle u_{\tau}^{\prime} u_{\eta}^{\prime}\right\rangle}{\tau_{w} / \rho}=1.0 \text {, }
$$

where $\tau, \eta$ and $\lambda$ are the wall tangential, normal and binormal coordinates, respectively.

\section{Boundary Conditions}

Periodic boundary conditions are used for open boundaries from left to right and from top to bottom, similar to Benhamadouche and Laurence [7]. Hassan and Barsamian [9], however, use the full depth of the tube bank (for the same data) because they believe that the largest turbulent length scale of the flow is large enough to require it. The mass flow is set at a given value to provide a flow through the flow domain. Although the mean velocity upstream of the tube bundle is reported to be 
$1.06 \mathrm{~m} / \mathrm{sec}$, the data reported for the mean streamwise velocity do not correspond to this velocity. A Bspline curve was fitted to the mean streamwise velocity data and numerically integrated using the midpoint rule on a fine grid. The data reported by $S \& B$ show a zero value for $U$ at $(x, y)=(0,0.012$ $\mathrm{m})$. Actually, the radius of the tube is $0.01085 \mathrm{~m}$. The B-spline curve is set to zero at $0.01085 \mathrm{~m}$, with a sharp increase, which corresponds to the DNS simulations of the same flow shown by Ref. [7]. The mass flow used for the periodic boundary conditions in the streamwise direction is based on the mass flow computed from the numerical integration of the B-spline curve. This value is $40.75 \mathrm{~kg} / \mathrm{sec}$, based on a water density of $998.2 \mathrm{~kg} / \mathrm{m}^{3}$, an area of $(0.045-.0217) \mathrm{m}$ and an average velocity of 1.752 $\mathrm{m} / \mathrm{sec}$.

\section{Application of Best Practice Guidelines}

The Journal of Fluids Engineering (JFE) published by the American Society of Mechanical Engineers (ASME) has developed criteria ${ }^{\mathrm{b}}$ for numerical accuracy for numerical-related articles submitted to the journal. The criteria are referred to as 'The Statement of Numerical Accuracy'. This statement has been used as a best practice guideline for the present simulation. These guidelines are:

1. The basic features of the method including formal truncation error of individual terms in the governing numerical equations must be described.

2. Methods must be at least second order accurate in space.

3. Inherent or artificial viscosity (or diffusivity) must be assessed and minimized.

4. Grid independence or convergence must be established.

5. When appropriate, iterative convergence must be addressed.

6. In transient calculations, phase error must be assessed and minimized.

7. The accuracy and implementation of boundary and initial conditions must be fully explained.

8. An existing code must be fully cited in easily available references.

9. Benchmark solutions may be used for validation for a specific class of problems.

10. Reliable experimental results may be used to validate a solution.

FLUENT is employed using 2D double precision, running on a 12-cpu Sun 4800 workstation. FLUENT uses the finite volume method. The discretization of the terms is given in the FLUENT Users' Manual. The viscous terms are discretized using a centered difference scheme ( $2^{\text {nd }}$ order). The pressure term is evaluated using a centered scheme. The discretization scheme for the convective terms is specified by the user. The $2^{\text {nd }}$ order [15] QUICK scheme is used herein. There is no added, only inherent numerical viscosity present in the discretization scheme. This error is minimized by using a fine enough mesh. The segregated solver is used to solve the discretized equations; the default under-relaxation factors are used. The SIMPLE algorithm is used for pressure-velocity coupling.

The issue of iterative convergence was investigated by the tolerance levels for equation residuals. The default value for convergence in FLUENT is $1.0 \times 10^{-3}$. The solution for steady flow in the tube bank was calculated for convergence tolerances of $1.0 \times 10^{-3}$ to $1.0 \times 10^{-6}$ and is shown in Figure $4 \mathrm{a}$. As shown, a tolerance of $1.0 \times 10^{-5}$ is sufficient to converge the solution; however, residual tolerances are set to $1.0 \times 10^{-6}$. Figure $4 \mathrm{~b}$ illustrates the ensemble-averaged streamwise velocity $\langle u\rangle$ at two points in the flow as functions of time step. The time step is clearly small enough to capture the large scale motions without unphysical oscillations. Figure 5 illustrates two grids that were used to check for grid independence. The maximum difference for the mean streamwise velocity using the finer gird, with 59,904 cells, Fig. 5b, was $4.9 \%$ relative to the grid with 14,976 cells, Fig. 5 a, for a two-equation turbulence model. The grid shown in Fig. 5a is used for all of the results reported herein.

\footnotetext{
${ }^{\mathrm{b}}$ http://journaltool.asme.org/Templates/JFENumAccuracy.pdf
} 


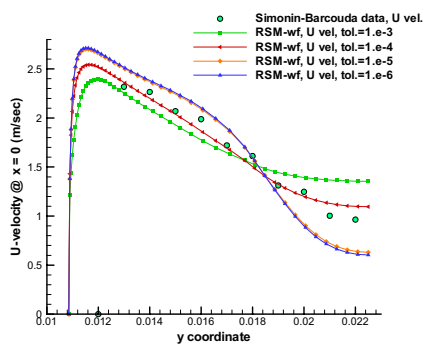

(a)

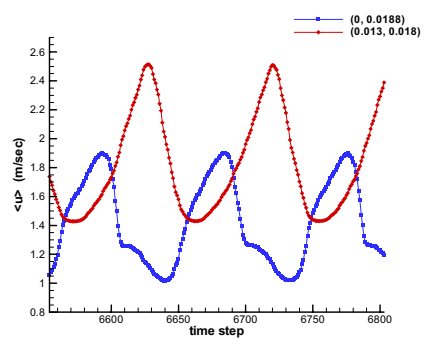

(b)

Figure 4. (a) Results for the iterative convergence study. (b) Time plots of the ensemble average velocity at two points in the flow for 250 steps.

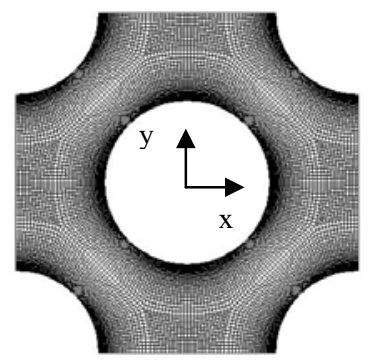

(a)

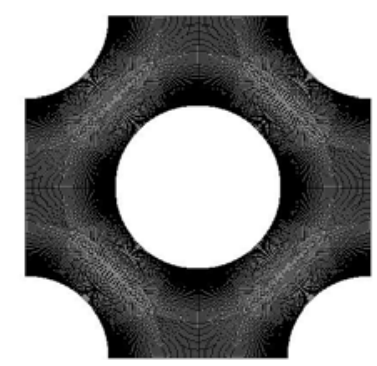

(b)

Figure 5. Two grids used in the grid independent study having (a) 14, 976 cells and (b) 59,904 cells. Grid (a) is used for the results reported below and defines the coordinates. The tubes have diameter of $0.0217 \mathrm{~m}$ and are spaced $0.0225 \mathrm{~m}$ apart in both directions.

For the URANS calculations, the second-order implicit time differencing scheme was employed. The time step was set to be adaptive with the minimum time step set to $1 \times 10^{-5} \mathrm{sec}$ and the maximum set to $5 \times 10^{-3}$; the time step size was usually between 2 and $5 \times 10^{-4} ; 5000$ time steps were computed for the present results. The results were compared with varying numbers of time steps to ensure that the time-averaged results are unchanging. A time step required about 22 seconds on the Sun 4800 . The default truncation error tolerance of 0.01 for the time step was used. It was found that if the residual tolerance was set to $1 \times 10^{-5}$ or $1 \times 10^{-6}$ and enough iterations at each time step allowed for convergence (1000), the simulation remained steady state. However, when the maximum number of iterations was set to 110 for each time step, with the residual tolerance set to $1 \times 10^{-6}$, it was found that the solution would converge every other time step or occasionally every third time step to the desired tolerance, while it would still converge to $1.5 \times 10^{-5}$ or lower on the other time steps. This approach apparently allowed there to be vortex shedding, due most likely to a numerical 'kick' caused by the uneven convergence, though the convergence is adequate according to the iterative convergence test described earlier. It is not unusual that a numerical perturbation is required to initiate unsteadiness in a simulation that is, in reality, an unsteady flow. Hence, it is believed that the above approach used for the unsteady flow calculation is reasonable and acceptable. Furthermore, the results for the unsteady calculations will be shown below to better match the S\&B data.

\section{Results and Discussion}


The S\&B data include results for the mean streamwise and transverse velocities, $U$ and $V$, the two normal stresses, $\overline{u^{\prime \prime}}$ and $\overline{v^{\prime \prime}}$, and the shear stress $\overline{u^{\prime \prime} v^{\prime \prime}}$ profiles at five locations. Comparisons are made at four locations: $\mathrm{x}=0,11 \mathrm{~mm}$ and $16.5 \mathrm{~mm}$, and $\mathrm{y}=0$. The $\mathrm{S} \& \mathrm{~B}$ data for the Reynolds stresses are given as constant and must therefore have been (long) time-averaged, such that they include the nonturbulent fluctuating components.

Figures 6-9 illustrate the computed results for the mean and fluctuating quantities at the above locations for the steady and unsteady RANS approaches compared to the data of Simonin and Barcouda. In the case of the URANS results, the long-time averaged or time-averaged ensembleaveraged results are plotted. For the fluctuating quantities, the contributions for both the coherent and turbulent fluctuations are each shown along with their sums. It can be seen that the results for URANS for the mean velocity $U$ are very close to the data for $\mathrm{x}=0,11$ and 16.5 and close for $\mathrm{y}=0$. The results for steady RANS are clearly inferior to the URANS results compared to the S\&B data. The present 2D URANS RSM results for $U$ are comparable to 3D URANS RSM results of Benhamadouche and Laurence [7] (hereafter referred to as B\&L), except that the present results are superior for $U$ at $\mathrm{y}=0$. The RSM of $\mathrm{B} \& \mathrm{~L}$ is somewhat different from the present one; the $\mathrm{B} \& \mathrm{~L}$ version does not use the pressure reflection term, nor does it employ the eddy viscosity in the dissipation rate equation as does FLUENT. Also, the grid of B\&L is slightly coarser. B\&L claim that $3 \mathrm{D}$ calculations are necessary, and give $2 \mathrm{D}$ results that are quite inferior to their $3 \mathrm{D}$ results.

The mean velocity $V$ data at $\mathrm{x}=0$ are actually two profiles, one taken above the center cylinder and one below [15], then drawn mirrored. The differences in the two profiles is an indication of the uncertainty in the measurements at this location. The present $2 \mathrm{D}$ computations are quite close to the far more costly 3D URANS, LES and DNS calculations reported in B\&L. The present results for $V$ at $\mathrm{x}=11 \mathrm{~mm}$ and $16.5 \mathrm{~mm}$ are close to the S\&B data for both the RANS and the URANS approaches. At $\mathrm{y}=0$, symmetry arguments require $V$ to be zero; the calculations are quite close to zero.

For the normal stress $\overline{u^{\prime \prime}}$, the URANS results are poorer than the RANS results and show large departures from the $R \& B$ data. It is also the case that the computed results of $B \& L$ for $\overline{u^{\prime \prime}}$ show significantly better agreement with $R \& B$ than the present results. However, for $\overline{v^{\prime \prime 2}}$, the URANS calculations are all significantly better than for the RANS calculations, though the magnitude of the maximum in each case is not as high as the $R \& B$ data. The importance of the contribution of the coherent fluctuations is clearly shown in the figures. The present results for the normal stresses are not as good as for B\&L's 3D results, but range from the same to far better than the $2 \mathrm{D}$ B\&L results. Finally, results for the shear stress $\overline{u^{\prime \prime} v^{\prime \prime}}$ are better than or about the same for both unsteady and steady results and agree quite well with the S\&B data; the shear stress should be zero for $\mathrm{y}=0$ based on symmetry. The most important fluctuating stress is the shear stress because it represents transport of momentum in an orthogonal direction from the momentum equation in question, whereas the normal stresses could actually be combined with the pressure term to represent a modified pressure. (The normal stresses appear with a gradient in the same direction as the pressure.)

\section{Conclusions}

The present 2D URANS results for the (long-time averaged) mean velocities for the tube-bank flow are quite satisfactory relative to the S\&B data, especially the streamwise velocity, and show correct trends. A primary reason for this is that the flow has been correctly computed as a nonstationary flow. The shear stress results are also satisfactory and show correct trends. While predictions for the normal stresses are not always satisfactory, they appear to have a relatively small effect on the results for the mean velocities. 


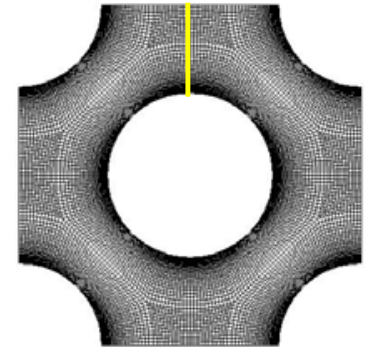

(a)

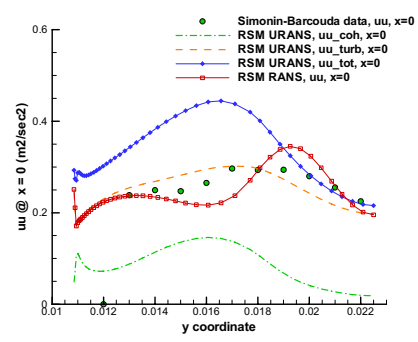

(d)

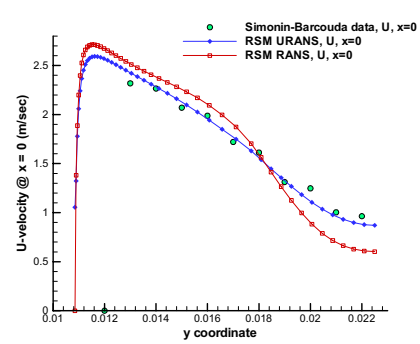

(b)

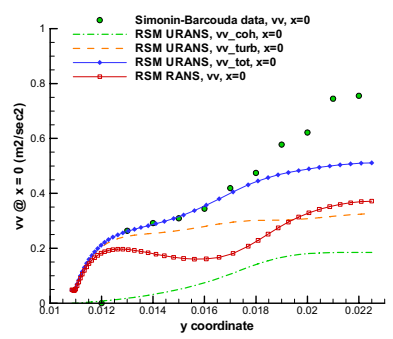

(e)

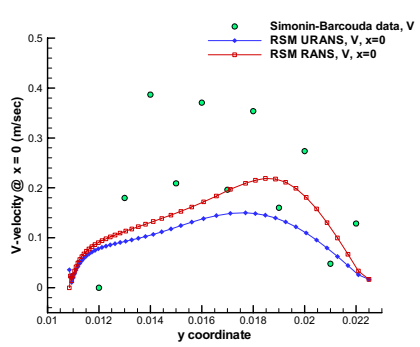

(c)

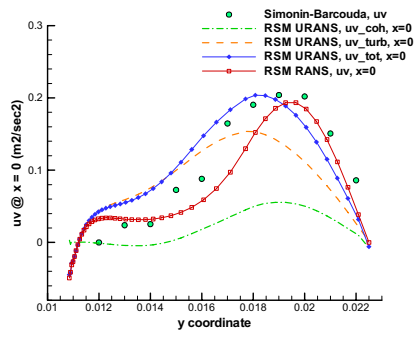

(f)

Figure 6. RANS and URANS results at (a) $\mathrm{x}=0$ for (b) $\mathrm{U}$ and (c) V mean velocities; and (d) $\overline{u^{\prime \prime}}$, (e) $\overline{v^{\prime \prime}}$ and (f) $\overline{u^{\prime \prime} v^{\prime \prime}}$ correlations for coherent, turbulent and total fluctuations compared to data [3].

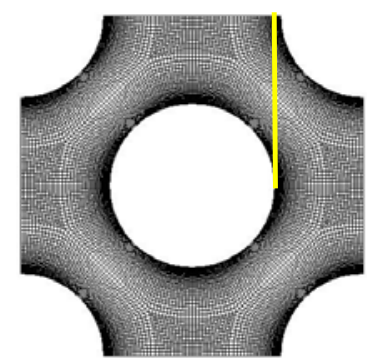

(a)

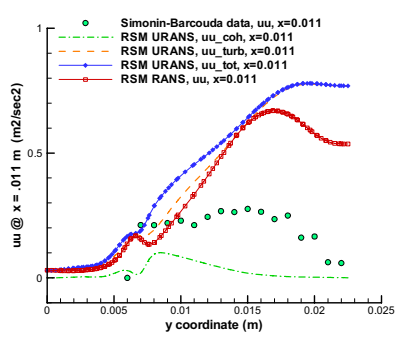

(d)

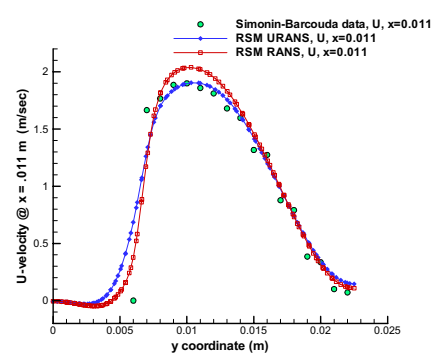

(b)

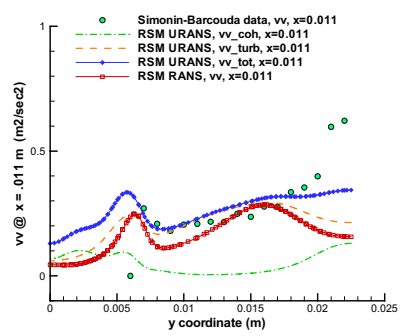

(e)

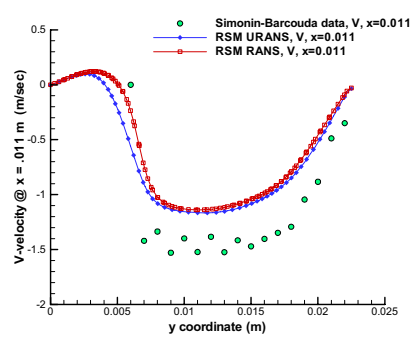

(c)

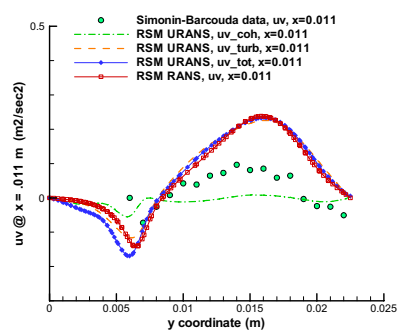

(f)

Figure 7. RANS and URANS results at (a) $\mathrm{x}=11 \mathrm{~mm}$ for (b) U and (c) V mean velocities; and (d) $\overline{u^{\prime \prime}}$, (e) $\overline{v^{\prime \prime}}$ and (f) $\overline{u^{\prime \prime} v^{\prime \prime}}$ correlations for coherent, turbulent and total fluctuations compared to data [3]. 


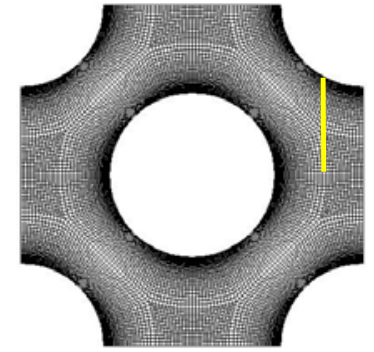

(a)

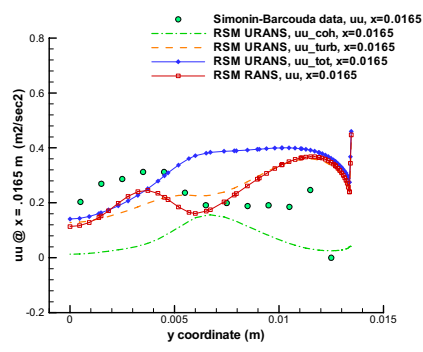

(d)

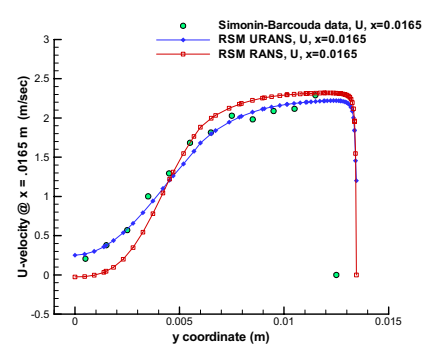

(b)

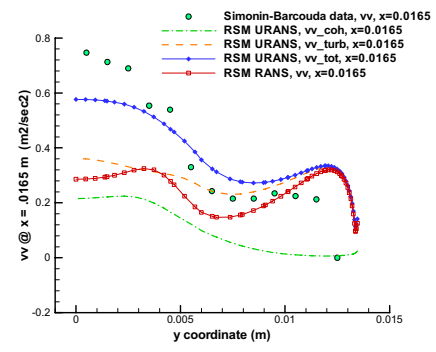

(e)

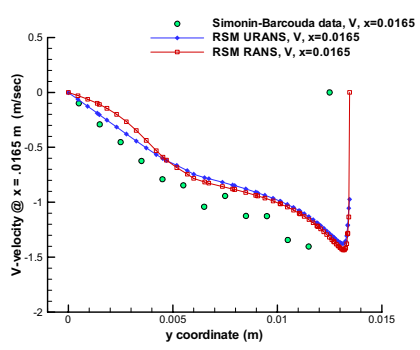

(c)

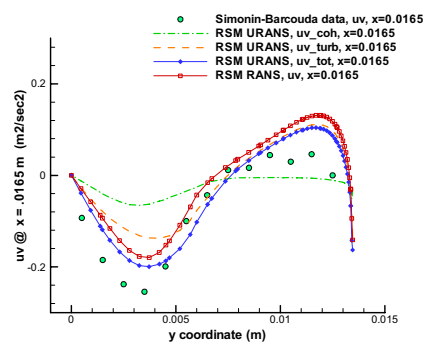

(f)

Figure 8. RANS and URANS results at (a) $\mathrm{x}=16.5 \mathrm{~mm}$ for (b) $\mathrm{U}$ and (c) V mean velocities; and (d) $\overline{u^{\prime \prime 2}}$, (e) $\overline{v^{\prime \prime 2}}$ and (f) $\overline{u^{\prime \prime} v^{\prime \prime}}$ correlations for coherent, turbulent and total fluctuations compared to data [3].

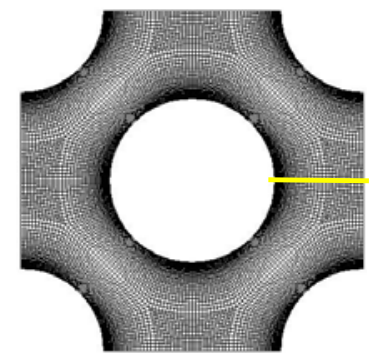

(a)

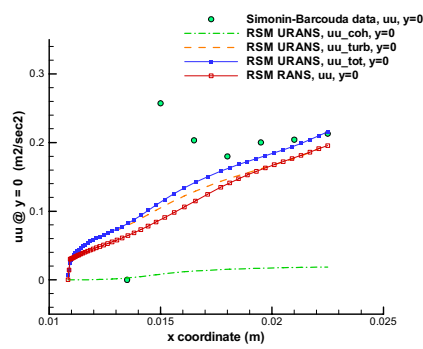

(d)

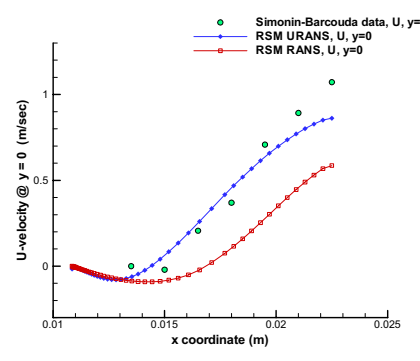

(b)

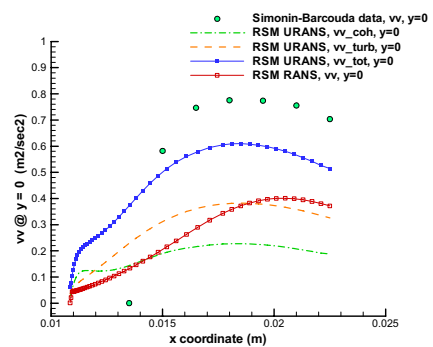

(e)

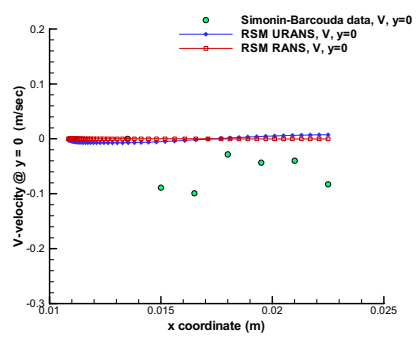

(c)

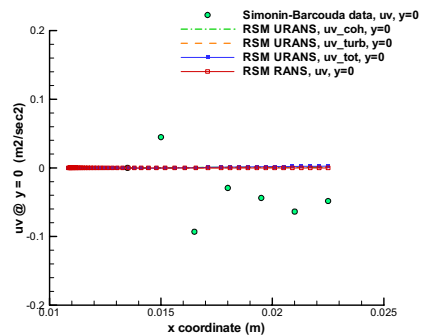

(f)

Figure 9. RANS and URANS results at (a) $\mathrm{y}=0$ for (b) $\mathrm{U}$ and (c) V mean velocities; and (d) $\overline{u^{\prime \prime 2}}$, (e) $\overline{v^{\prime \prime}}$ and (f) $\overline{u^{\prime \prime} v^{\prime \prime}}$ correlations for coherent, turbulent and total fluctuations compared to data [3]. 


\section{Acknowledgements}

Work supported by the U.S. Department of Energy, Office of Nuclear Energy, under DOE Idaho Operations Office Contract DE-AC07-05ID14517.

\section{References}

[1] MacDonald, P. E., J. W. Sterbentz, R. L. Sant, P. D. Bayless, R. R. Schultz, H. D. Gougar, R. L. Moore, A. M. Ougouag, and W. K. Terry, NGNP Preliminary Point Design, Results of the Initial Neutronics and Thermal-hydraulic Assessments. Tech. Report INEEL/EXT-03-00870 Rev. 1.

[2] General Atomics, "Gas Turbine - Modular Helium Reactor (GT-MHR) Conceptual Design Description Report," Doc. 910720, Rev. 1, July, 1996.

[3] Simonin, O., and Barcouda, M., "Measurements of Fully Developed Turbulent Flow across Tube Bundle," Proceedings of the Third International Symposium on Applications of Laser Anemometry to Fluid Mechanics, Lisbon, Portugal, 1986, pp. 21.5.1-21.5.5.

[4] Simonin, O., and Barcouda, M., "Measurements and prediction of turbulent flow entering a staggered tube bundle," in: 4th International Symposium on Applications of Laser Anemometry to Fluid Mechanics, Lisbon, Portugal, paper 5.23, 1988

[5] ERCOFTAC-IAHR workshop, 2nd ERCOFTAC-IAHR Workshop on Refined Flow Modelling, Lisbon, Portugal, 1993.

[6] ERCOFTAC-IAHR workshop, 3rd ERCOFTAC-IAHR Workshop on Refined Flow Modelling, UMIST, Manchester, United Kingdom, 1994.

[7] Benhamadouche, S. and Laurence, D., "LES, coarse LES, and transient RANS comparisons on the flow across a tube bundle," International Journal of Heat and Fluid Flow, 24(4), 2003, 470479.

[8] Rollet_Miet, P.; Laurence, D. and Ferziger, J., "LES and RANS of turbulent flow in tube bundles," International Journal of Heat and Fluid Flow, 20(3), 1999, 241-254.

[9] Hassan, Y. A., Barsamian, H. R., "Tube bundle flows with the large Eddy simulation technique in curvilinear coordinates," International Journal of Heat and Mass Transfer, 47, 2004, 3057-3071.

[10] Moulinec, C., Hunt, J. C. R. and Nieuwstadt, F. T. M., "Disappearing wakes and dispersion in numerically simulated flows through tube bundles," Flow, Turbulence and Combustion, 73(2), 2004, 95-116.

[11] FLUENT, version 6.2.16, FLUENT Inc., 10 Cavendish Court, Centerra Resource Park, Lebanon, NH, 03766, 2005.

[12] Wilcox, D. C., "Turbulence Modeling for CFD," DCW Industries, Inc., La Cañada, CA, 1994.

[13] Hanjalic, K., "Will RANS survive LES? A View of Perspectives," Journal of Fluids Engineering, 127, 831-839, 2005.

[14] Laurence, D. personal communication, Mar. 2005.

[15] Johnson, R. W. and MacKinnon, R. J., "Equivalent versions of the QUICK scheme for finitedifference and finite-volume numerical methods," Communications in Applied Numerical Methods, 8(12), 841-848, 1992. 